\title{
An SPM8-Based Approach for Attenuation Correction Combining Segmentation and Nonrigid Template Formation: Application to Simultaneous PET/MR Brain Imaging
}

\author{
David Izquierdo-Garcia ${ }^{1}$, Adam E. Hansen ${ }^{2}$, Stefan Förster ${ }^{3}$, Didier Benoit ${ }^{2}$, Sylvia Schachoff ${ }^{3}$, Sebastian Fürst $^{3}$, \\ Kevin T. Chen ${ }^{1,4}$, Daniel B. Chonde ${ }^{1,4,5}$, and Ciprian Catana ${ }^{1}$ \\ ${ }^{I}$ Athinoula A. Martinos Center for Biomedical Imaging, Department of Radiology, Massachusetts General Hospital and Harvard \\ Medical School, Charlestown, Massachusetts; ${ }^{2}$ Department of Clinical Physiology, Nuclear Medicine, and PET, Rigshospitalet, \\ Copenhagen University Hospital, Copenhagen, Denmark; ${ }^{3}$ Department of Nuclear Medicine, Technische Universität München, \\ Munich, Germany; ${ }^{4}$ Department of Health Sciences and Technology, Massachusetts Institute of Technology, Cambridge, \\ Massachusetts; and ${ }^{5}$ Program in Biophysics, Harvard University, Cambridge, Massachusetts
}

We present an approach for head MR-based attenuation correction (AC) based on the Statistical Parametric Mapping 8 (SPM8) software, which combines segmentation- and atlas-based features to provide a robust technique to generate attenuation maps ( $\mu$ maps) from MR data in integrated PET/MR scanners. Methods: Coregistered anatomic MR and CT images of 15 glioblastoma subjects were used to generate the templates. The MR images from these subjects were first segmented into 6 tissue classes (gray matter, white matter, cerebrospinal fluid, bone, soft tissue, and air), which were then nonrigidly coregistered using a diffeomorphic approach. A similar procedure was used to coregister the anatomic MR data for a new subject to the template. Finally, the CT-like images obtained by applying the inverse transformations were converted to linear attenuation coefficients to be used for AC of PET data. The method was validated on 16 new subjects with brain tumors $(n=12)$ or mild cognitive impairment $(n=4)$ who underwent $\mathrm{CT}$ and PET/MR scans. The $\mu$ maps and corresponding reconstructed PET images were compared with those obtained using the gold standard CT-based approach and the Dixon-based method available on the Biograph mMR scanner. Relative change $(\mathrm{RC})$ images were generated in each case, and voxel- and regionof-interest-based analyses were performed. Results: The leaveone-out cross-validation analysis of the data from the 15 atlasgeneration subjects showed small errors in brain linear attenuation coefficients (RC, $1.38 \% \pm 4.52 \%$ ) compared with the gold standard. Similar results (RC, $1.86 \% \pm 4.06 \%)$ were obtained from the analysis of the atlas-validation datasets. The voxel- and region-of-interestbased analysis of the corresponding reconstructed PET images revealed quantification errors of $3.87 \% \pm 5.0 \%$ and $2.74 \% \pm$ $2.28 \%$, respectively. The Dixon-based method performed substantially worse (the mean RC values were $13.0 \% \pm 10.25 \%$ and $9.38 \%$ $\pm 4.97 \%$, respectively). Areas closer to the skull showed the largest improvement. Conclusion: We have presented an SPM8-based approach for deriving the head $\mu$ map from MR data to be used for PET AC in integrated PET/MR scanners. Its implementation is straightforward and requires only the morphologic data acquired with a single MR sequence. The method is accurate and robust,

Received Dec. 12, 2013; revision accepted Aug. 4, 2014.

For correspondence or reprints contact: Ciprian Catana, Athinoula A. Martinos Center for Biomedical Imaging, 149, 13th St., Room 1116, Charlestown, MA 02129.

E-mail: ccatana@nmr.mgh.harvard.edu

Published online Oct. 2, 2014.

COPYRIGHT (c) 2014 by the Society of Nuclear Medicine and Molecular Imaging, Inc. combining the strengths of both segmentation- and atlas-based approaches while minimizing their drawbacks.

Key Words: integrated PET/MRI; attenuation correction; segmentation; template

J Nucl Med 2014; 55:1825-1830

DOI: 10.2967/jnumed.113.136341

$\mathbf{A}_{\text {ttenuation correction (AC) is one of the biggest challenges }}$ in achieving accurate PET quantification in combined PET/MR imaging $(1,2)$. The goal of MR-based AC techniques is to address the challenge of converting the MR signal, related to the proton density and MR relaxation times of tissues, into linear attenuation coefficients (LACs), related to electron density, at PET energy levels (3).

The MR AC strategies can be broadly grouped into 3 classes: atlasbased approaches, segmentation-based approaches, and techniques aimed at simultaneously estimating the emission and attenuation data. In atlas-based methods, several pairs of coregistered MR and attenuation maps ( $\mu$ maps) are combined to form a template set (4-8). Any new subject's MR image is first coregistered to the MR template. The matched $\mu$ map from the atlas is then warped back into the subject's space using the inverse transformation. These methods require matched MR and transmission maps (or CT images), which are not always available, and accurate inter- and intrasubject coregistration between the training datasets. Local anatomic mismatches from the template, due to either natural or disease-related anatomic variants (e.g., tissue remodeling or surgical procedures), tend to introduce biased estimations, which are difficult to address (2). Segmentation-based approaches involve segmentation of morphologic MR images into tissue classes to which approximate LACs are subsequently assigned (9-13). Local anatomic variations are usually better addressed on segmentationbased approaches; however, errors are introduced because of, among other factors, the inability of these methods to classify all the different tissue classes and the use of a handful of averaged LACs that may not represent accurately the subject-specific tissue properties $(2,13)$. Finally, the simultaneous emission and $\mu$-map estimation is based on iterative methods following maximum a posteriori schemes with different correction terms (penalties) (14-17). Such approaches usually require time-of-flight information and 


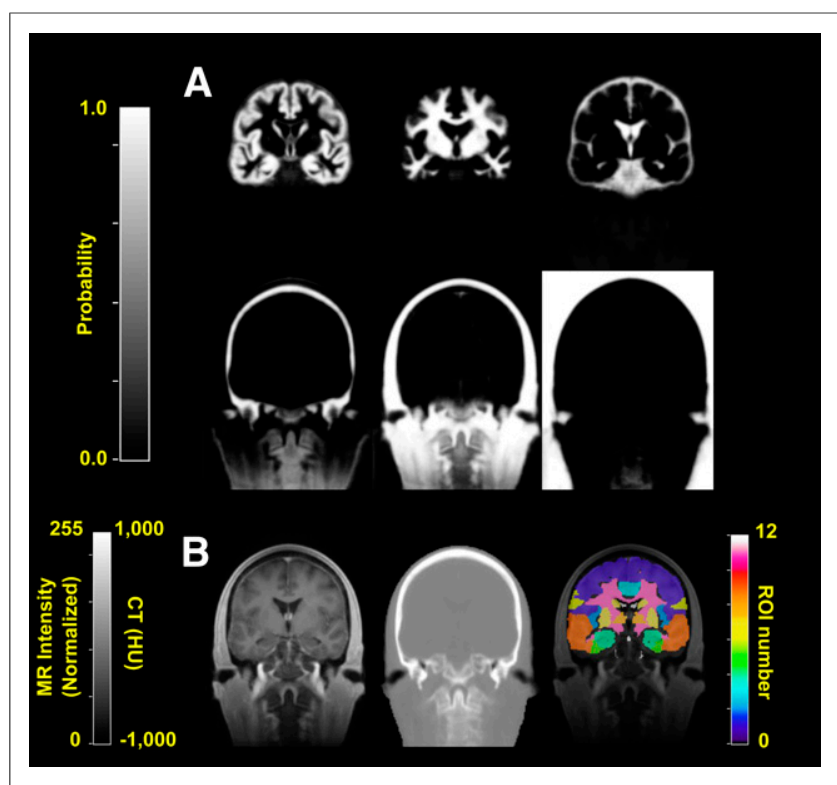

FIGURE 1. Template images generated for atlas method. (A) Tissue classes at final iteration of diffeomorphic nonrigid coregistration method (DARTEL). From left to right are gray matter, white matter, and cerebrospinal fluid (top row) and bone, soft tissue, and air (bottom row). (B) Template MR (left) and CT (middle) images and Automatic Anatomic Labeling regions (right) used for $\mathrm{ROI}$ analysis overlaid on MR template image.

determine the attenuation sinogram up to a constant offset $(2,16$, 18). Because the estimation of the LACs from the emission data is a "very ill-posed problem" (16), the iterative solution is highly dependent on both the initialization and the penalty terms introduced and could potentially lead to bias and inaccurate estimation $(16,18)$. Furthermore, in the case of tracers with very specific uptake, the PET emission data may not provide complete information to estimate accurate LACs in all the voxels (16). New transmission-based techniques have recently been redesigned to allow simultaneous emission and transmission acquisition (19). However, they involve additional radiation exposure and require time-of-flight detector capabilities to simultaneously detect the events from the subject and the transmission source.

We present an approach for MR AC based on the widely available Statistical Parametric Mapping 8 software (SPM8; Wellcome Trust Centre for Neuroimaging, University College London) that allows accurate AC for brain PET studies. Our approach combines the strengths of the segmentation- and atlas-based approaches (e.g., use of accurate segmentation of tissue classes to enable precise nonrigid coregistration to form a template), which in principle could help minimize some of their specific drawbacks. The technique has been successfully tested on datasets acquired at 2 different institutions using integrated PET/MR scanners (Biograph mMR; Siemens Healthcare). The performance of the method was compared with both that of the AC method provided by the manufacturer (the Dixon-based segmented method (10)) and that of the gold standard for PET/CT imaging (CT-based AC).

\section{MATERIALS AND METHODS}

\section{Image Acquisition}

Atlas Generation Data. CT and MR images of 15 subjects diagnosed with glioblastoma were retrospectively analyzed to create the template. The MR images were acquired on a 3-T MAGNETOM Trio (Siemens
Healthcare). T1-weighted 3-dimensional magnetization-prepared rapid gradient echo (MPRAGE) MR images were acquired after administration of an MR contrast agent (Magnevist [gadopentetate dimeglumine]; Bayer HealthCare) (details are provided in the supplemental materials, available at http://jnm.snmjournals.org).

All subjects underwent CT examinations within 1 mo of their MR imaging scans (LightSpeed QX/I; GE Healthcare). No surgical interventions were performed in the interval between the $\mathrm{CT}$ and MR imaging sessions. All subjects gave written informed consent, and the local Institutional Review Board approved the study.

Atlas Validation Data. Sixteen CT and PET/MR datasets from subjects diagnosed with different grades of brain tumor $(n=12)$ or mild cognitive impairment $(n=4)$ were retrospectively included in the study and became the validation dataset. These data were collected at Copenhagen University Hospital, Denmark $(n=7)$, and Technische Universität München, Germany $(n=9)$. The PET and MR images were acquired simultaneously using the Biograph mMR scanner (20). All subjects gave written informed consent, and the local Institutional Review Boards approved the study.

The emission data were acquired $74.5 \pm 19.9$ min (mean $\pm \mathrm{SD})$ after administration of $199.5 \pm 25.4 \mathrm{MBq}$ of ${ }^{18} \mathrm{~F}-\mathrm{FDG}(n=9)$ or ${ }^{18} \mathrm{~F}-O$ - $\left(2-{ }^{18} \mathrm{~F}\right.$-fluoroethyl)-L-tyrosine (FET) $(n=7)$. Data were acquired in 3-dimensional mode for $15 \mathrm{~min}(16.8 \pm 2.19 \mathrm{~min})$ and were reconstructed using the 3-dimensional ordered-subsets expectation maximization algorithm with 3 iterations and 21 subsets, with corrections for random coincidences, variable detector sensitivity, dead time, isotope decay, scatter, and photon attenuation. Images were reconstructed into a $344 \times 344 \times 127$ matrix with voxel sizes of $2.09 \times 2.09 \times 2.03 \mathrm{~mm}$ $(n=9)$ and $0.835 \times 0.835 \times 2.03 \mathrm{~mm}(n=7)$.

MR data were acquired simultaneously with the PET data using MPRAGE and dual-echo Dixon volume-interpolated breath-hold examination (VIBE) sequences. Low-dose CT images were acquired at both sites without contrast material for all subjects on the day of the PET/MR sessions using a Biograph 64 scanner (Siemens) (the supplemental material provides data acquisition details).

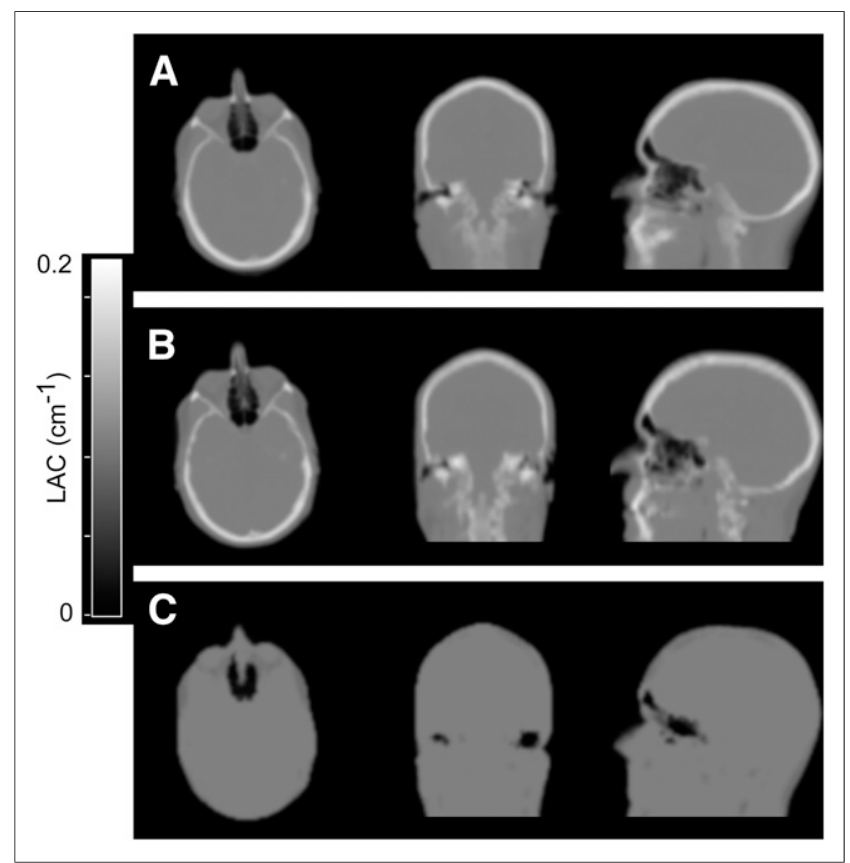

FIGURE 2. Comparison of attenuation maps for representative atlasvalidation subject. $\mu_{\text {atlas }}(\mathrm{A}), \mu_{\mathrm{CT}}(\mathrm{B})$, and $\mu_{\text {Dixon }}(\mathrm{C})$ are displayed in 3 orientations. 


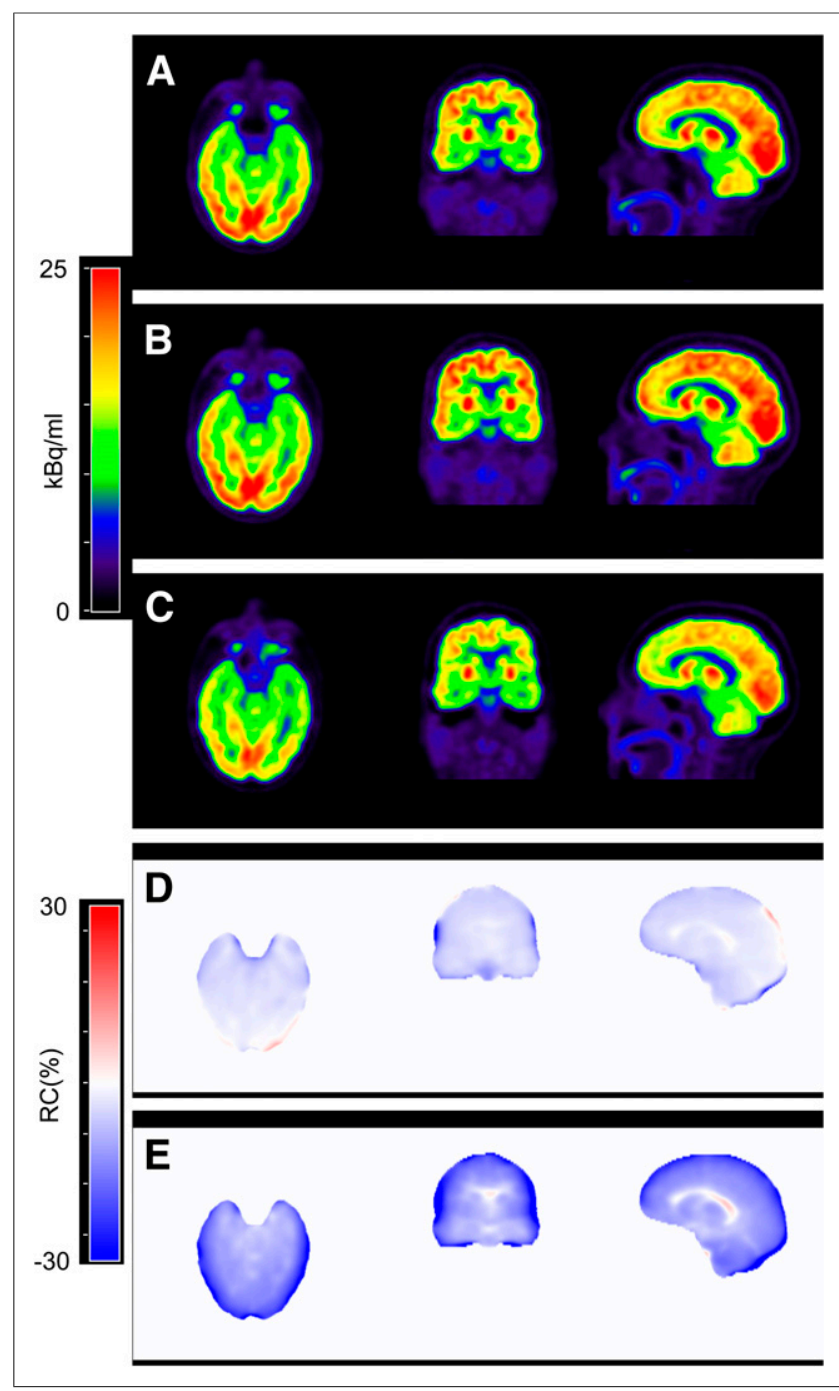

FIGURE 3. (A-C) Comparison of PET images reconstructed with the $3 \mathrm{AC}$ methods for 1 validation subject: $\mathrm{PET}_{\text {atlas }}(\mathrm{A}), \mathrm{PET}_{\mathrm{CT}}(\mathrm{B})$, and $\mathrm{PET}_{\text {Dixon }}(\mathrm{C})$. ( $\mathrm{D}$ and $\left.\mathrm{E}\right)$ Corresponding $\mathrm{RC}$ images (masked to highlight only brain area) for PET data reconstructed with atlas $A C(D)$ and Dixon $A C(E)$ with respect to CT AC method.

\section{MR and CT Image Preprocessing}

All MR images were intensity-normalized following a previously described method (21) using FreeSurfer, version 5.2 (http://surfer.nmr. mgh.harvard.edu), to allow better image segmentation and therefore a better template formation.

CT images were then coregistered to the MR images using the SPM8 affine automatic coregistration. Finally, CT images were resliced into the
MR (MPRAGE) space to allow for comparisons of the $\mu$-map generation and AC methods.

\section{Atlas Generation}

The template was formed by nonrigid coregistration of all 15 subjects from the atlas generation dataset. SPM8 was used to perform the 3 steps involved in the template formation: MR image segmentation into tissue classes, nonrigid coregistration of these tissue classes, and warping of the CT images into the same (atlas) space (these steps are described in more detail in the supplemental materials). The final CT template was created by averaging all the warped CT images.

\section{Atlas Validation}

To validate the atlas generation methodology, we used the leave-oneout cross-validation approach with the 15 datasets used for constructing the template.

The final template (generated from all 15 datasets) was further validated using the 16 validation subjects. To obtain an atlas-based CT image for a new subject, the tissue classes derived from the intensity-normalized MR image were warped into the template space and then the final CT template was inversely warped into the subject space. This was achieved by following the same 3 steps used in the atlas generation procedure: MR image segmentation (SPM8 New Segment), nonrigid coregistration of the tissue classes to the previously created template (SPM8 DARTEL [Diffeomorphic Anatomical Registration Through Exponentiated Lie Algebra] Existing Template), and finally inverse warping of the final CT template image into the subject space (SPM8 Create Inverse Warped). This final image becomes the image used for the atlas-based AC method.

\section{AC}

To compare our AC method with the CT AC, the Hounsfield units of the atlas-based and resliced CT images generated from the validation subjects were converted to LACs using the bilinear transformation (22) to generate the corresponding $\mu$ maps: $\mu_{\text {atlas }}$ and $\mu_{\mathrm{CT}}$, respectively. Gaussian smoothing with a 4-mm kernel was applied to these $\mu$ maps to match the PET spatial resolution. Additionally, the $\mu$ maps generated using the method currently available on the Biograph mMR scanner $\left(\mu_{\text {Dixon }}\right)(10)$ were also available for comparison. Briefly, in the case of head imaging, the images acquired with the Dixon VIBE sequence are segmented into 3 tissue classes: air, fat, and soft tissue. LACs of 0 , 0.0854 , and $0.1000 \mathrm{~cm}^{-1}$, respectively, are then assigned to these tissue classes. Bone tissue is not segmented using this method. AC factors in sinogram space were generated from all these $\mu$ maps and used to correct the PET data for the validation subjects. The resulting PET volumes were named $\mathrm{PET}_{\text {atlas }}, \mathrm{PET}_{\mathrm{CT}}$, and $\mathrm{PET}_{\text {Dixon }}$, corresponding to the atlas-, CT- and Dixon-based AC methods, respectively.

\section{Image Analysis}

Voxel- and region-based analyses were performed to assess the accuracy of the $\mu$ maps and reconstructed PET images. Only voxels included in the head mask created by performing binary and morphologic operations (supplemental materials) on each subject's intensity-normalized

TABLE 1

Summary of ROI- and Voxel-Based Analysis Changes for Whole-Brain Area

\begin{tabular}{|c|c|c|c|c|c|}
\hline Analysis & Mean RC & $\mu_{\text {atlas }}$ & $\mu_{\text {Dixon }}$ & $\mathrm{PET}_{\text {atlas }}$ & $\mathrm{PET}_{\text {Dixon }}$ \\
\hline \multirow[t]{2}{*}{ ROI-based } & Absolute & $0.99 \pm 1.81[0.192 .76]$ & $3.04 \pm 3.15$ [1.99 4.51] & $2.74 \pm 2.28$ [1.54 5.42] & $9.38 \pm 4.97\left[\begin{array}{ll}4.44 & 16.16\end{array}\right]$ \\
\hline & Nonabsolute & $0.30 \pm 1.91[-0.131 .42]$ & $0.45 \pm 3.62[-2.242 .19]$ & $-1.01 \pm 2.5[-2.25-0.02]$ & $-9.03 \pm 5.6[-16.16-1.89]$ \\
\hline \multirow[t]{2}{*}{ Voxel-based } & Absolute & $1.86 \pm 4.06$ & $4.18 \pm 6.68$ & $3.87 \pm 5.0$ & $13.0 \pm 10.25$ \\
\hline & Nonabsolute & $0.49 \pm 4.44$ & $-1.35 \pm 7.76$ & $-1.18 \pm 6.21$ & $-12.74 \pm 10.57$ \\
\hline
\end{tabular}

Mean RC values were obtained by averaging across all subjects. Data are percentages. Data in brackets are minimum and maximum. 


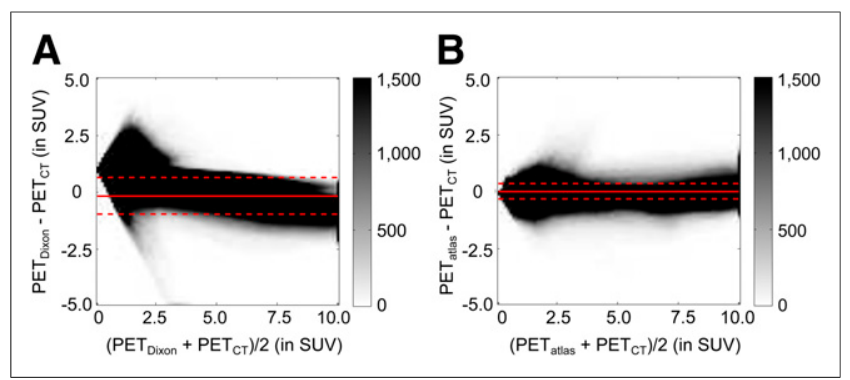

FIGURE 4. Bland-Altman plots showing voxel-based comparisons (for all validation subjects) between $\mathrm{PET}_{\text {Dixon }}$ and $\mathrm{PET}_{\mathrm{CT}}(\mathrm{A})$ and between $\mathrm{PET}_{\text {atlas }}$ and $\mathrm{PET}_{\mathrm{CT}}$ (B). Color bar shows density of voxels on histogram grid.

MR image head mask were used for comparisons. Bland-Altman plots, bias, and variability Pearson correlation coefficients were calculated for all comparisons. Absolute RCs were defined as follows:

$$
R C_{I_{X}}(\%)=100 \cdot \frac{\left|I_{X}-I_{g s}\right|}{I_{g s}}
$$

where $X$ is the AC method (Dixon or atlas); $I_{X}$ corresponds to either the PET images $\left(\mathrm{PET}_{\text {Dixon }}\right.$ or $\left.\mathrm{PET}_{\text {atlas }}\right)$ or the $\mu$ maps $\left(\mu_{\text {Dixon }}\right.$ or $\left.\mu_{\text {atlas }}\right)$; and $I_{g s}$ corresponds to the gold standard image $\left(\mathrm{PET}_{\mathrm{CT}}\right.$ or $\mu_{\mathrm{CT}}$ for PET and $\mu$-map comparisons, respectively). Nonabsolute RCs were calculated similarly but without taking the absolute value of the difference.

For the region-based analysis, 10 gray matter regions of interest (ROIs) obtained from the Automatic Anatomic Labeling template (i.e., frontal, insula, cingulate, limbic, occipital, parietal, basal ganglia, thalamus, temporal, and cerebellum), 1 white matter ROI, and 1 cerebrospinal fluid ROI were selected to cover most of the brain $(>90 \%)$. Because the frontal region is more prone to errors because of its proximity to the sinuses, where bone and air segmentation is difficult, the frontal ROI was further segmented into 28 Automatic Anatomic Labeling subregions for detailed analysis. A brain mask obtained from the subject-specific gray matter, white matter, and cerebrospinal fluid tissue probability maps was derived to calculate the voxel-based absolute differences. A brain cortex mask was additionally created for the validation dataset from the 5 outermost voxels of the brain mask (e.g., those adjacent to bone tissue) to assess the accuracy of the method in this challenging area.

Mean and maximum tumor-to-brain ratios (T/B) were calculated for all the ${ }^{18} \mathrm{~F}$-FET patients $(n=7)$ following a previously published method (23) (supplemental materials).

\section{Statistical Analysis}

The different methods were compared using paired Student $t$ tests. A $P$ value of less than 0.05 was considered to indicate statistical significance. Mean and SD percentage values for absolute RCs and nonabsolute RCs were computed across subjects. For ROI analysis, minimum and maximum percentage values for the average RCs across subjects were also calculated.

\section{RESULTS}

The tissue classes and template images generated using the iterative diffeomorphic image registration approach and the Automatic Anatomic Labeling ROIs used in this study are shown in Figure 1.

The leave-one-out cross-validation analysis of the 15 subjects included in the atlas generation dataset showed that the mean RC was $1.38 \% \pm 4.52 \%$ for all brain voxels and $0.75 \% \pm 1.6 \%$ for all ROIs (range, $0.16 \%-1.78 \%$ ).
Figure 2 shows $\mu$ maps obtained with the 3 methods for one of the validation subjects, demonstrating excellent correspondence between $\mu_{\text {atlas }}$ and $\mu_{\mathrm{CT}}$ and the obvious misclassification of bone tissues in the $\mu_{\text {Dixon }}$. The difference maps between the MR-based ( $\mu_{\text {atlas }}$ and $\mu_{\text {Dixon }}$ ) and CT-based $\mu$ maps are shown in Supplemental Figure 1. More detailed results of the ROI-based analysis are provided in Supplemental Figure 2.

A comparison of the reconstructed PET images corrected with the $3 \mathrm{AC}$ methods for one of the validation subjects is shown in Figure 3. As expected, the largest errors in the brain occurred in cortical areas, near the skull. The results of the quantitative ROIand voxel-based analyses are summarized in Table 1. Comparable results, in terms of tendency, were observed when either absolute or nonabsolute RCs were calculated (Table 1), with nonabsolute RCs showing much reduced biases in all cases. The errors in the 5 outermost voxels of the brain mask were $9.41 \% \pm 8.58 \%$ for $\mathrm{PET}_{\text {atlas }}$ and $28.15 \% \pm 13.36 \%$ for $\mathrm{PET}_{\text {Dixon }}$, similar to the underestimations previously reported in this area using the Dixon-based approach (24).

Figures $4 \mathrm{~A}$ and $4 \mathrm{~B}$ show the Bland-Altman plots for the voxelbased analyses for all validation subjects, comparing PET $_{\text {Dixon }}$ (Fig. 4A) and $\mathrm{PET}_{\text {atlas }}$ (Fig. 4B) with $\mathrm{PET}_{\mathrm{CT}}$.

The ROI-based comparison for the 12 ROIs is presented in Figure 5. Similar results were obtained in the 28 Automatic Anatomic Labeling frontal subregions (Supplemental Table 1).

The mean and maximum $\mathrm{T} / \mathrm{Bs}$ and standardized uptake values (SUVs) for the tumor and reference ROIs are given in Table 2. No significant differences were observed when mean and maximum T/B and SUV were compared between PET $_{\text {atlas }}$ and PET $\mathrm{PT}_{\mathrm{C}}$. Underestimation of mean and maximum $\mathrm{T} / \mathrm{B}$ on $\mathrm{PET}_{\text {Dixon }}$ just failed to reach statistical significance when compared with either $\mathrm{PET}_{\mathrm{CT}}$ or $\mathrm{PET}_{\text {atlas, }}$, whereas both mean and maximum SUV were significantly underestimated with $\mathrm{PET}_{\text {Dixon }}$ compared with either $\mathrm{PET}_{\mathrm{CT}}$ or $\mathrm{PET}_{\text {atlas }}$ (Table 2).

\section{DISCUSSION}

We have proposed and implemented a head MR AC approach for correcting the PET data acquired on integrated PET/MR scanners. In our approach, we combined features from both segmentation- and atlas-based techniques to implement a robust and flexible method for brain imaging. The method is based on the widely popular SPM software and uses the diffeomorphic image registration algorithm, DARTEL, as implemented in SPM8 $(25,26)$. The use of 6 segmented tissue classes (gray matter, white matter,

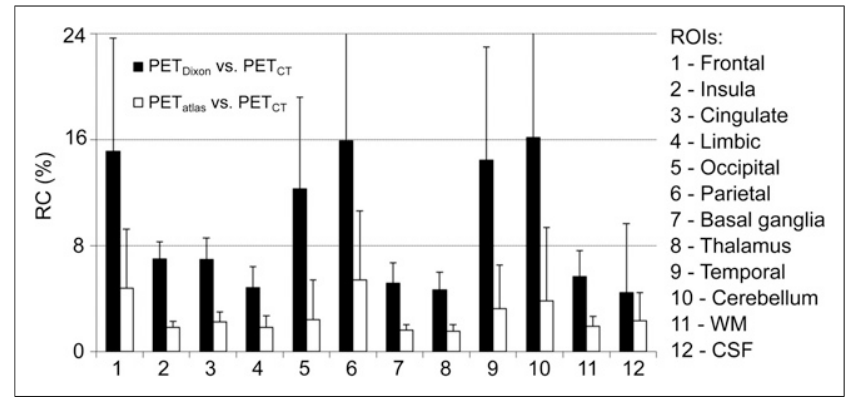

FIGURE 5. ROI-based analysis for all validation subjects of absolute RCs in PET images when using MR-based AC methods (PET atlas and $\left.\mathrm{PET}_{\text {Dixon }}\right)$ vs. $\mathrm{CT}$-based approach $\left(\mathrm{PET}_{\mathrm{CT}}\right) . \mathrm{CSF}=$ cerebrospinal fluid; $\mathrm{WM}=$ white matter. 
TABLE 2

Summary of T/B Results for ${ }^{18}$ F-FET Subset $(n=7)$

\begin{tabular}{lcccccc}
\hline \multicolumn{1}{c}{ Parameter } & PET $_{\mathrm{CT}}$ & $P, \mathrm{CT}$-atlas & PET $_{\text {atlas }}{ }^{*}$ & $P$, atlas-Dixon & PET $_{\text {Dixon }}{ }^{*}$ & $P, \mathrm{CT}_{-\mathrm{Dixon}}$ \\
\hline Mean SUV, tumor & $2.32 \pm 0.88$ & 0.28 & $2.28 \pm 0.92$ & $<0.003$ & $2.05 \pm 0.81$ & $<0.003$ \\
Maximum SUV, tumor & $3.69 \pm 1.97$ & 0.17 & $3.63 \pm 2.01$ & $<0.01$ & $3.27 \pm 1.78$ & $<0.006$ \\
Mean SUV, reference & $1.27 \pm 0.23$ & 0.7 & $1.26 \pm 0.24$ & $<0.002$ & $1.17 \pm 0.22$ & $<0.001$ \\
Mean T/B & $1.8 \pm 0.43$ & 0.16 & $1.77 \pm 0.45$ & 0.05 & $1.71 \pm 0.46$ & 0.07 \\
Maximum T/B & $2.82 \pm 1.14$ & 0.11 & $2.78 \pm 1.16$ & 0.05 & $2.7 \pm 1.16$ & 0.06 \\
\hline
\end{tabular}

${ }^{\star}$ Average across all ${ }^{18} \mathrm{~F}-\mathrm{FET}$ subjects.

cerebrospinal fluid, soft tissue, bone, and air) instead of the classic intensity-based MR images facilitates the nonrigid coregistration step: not only is more information available to perform the registration but the accuracy of the image segmentation enables better coregistration of the tissue classes. Because the DARTEL nonrigid coregistration method relies on these segmentations, our approach could in principle overcome local anatomic variants given appropriate segmentation and has the potential to perform more robustly than other purely atlas-based approaches in terms of both accuracy of LACs and adaptability to local anatomic features (3 examples of patients from the atlas-generation dataset with large tumors close to bone and with surgical procedures are shown in Supplemental Figs. 3-5). However, further studies in even more challenging subjects are required to fully demonstrate this potential advantage.

In terms of feasibility, our approach is easy to implement under MATLAB (MathWorks) and requires only free software (SPM and FreeSurfer) to generate a $\mu$ map in less than 30 min on a standard computer.

The similar results (in terms of RC) obtained for the postcontrast atlas generation and the precontrast validation datasets (Table 1) suggest that the use of contrast-enhanced MR images to generate the template does not affect the outcome. The differences between the mean RCs obtained from the analysis of the AC maps and the PET reconstructed images (Table 1) are due to the 3-dimensional cumulative effect that inaccurate $\mu$ maps have on the reconstructed images.

Some of the differences observed could be due to inaccuracies in the CT-derived $\mu$ map. Although currently accepted as the gold standard, CT AC can be affected by CT image artifacts, being one of the sources of errors in PET quantification (27). For example, artifacts commonly appear around metallic dental fillings, implants, and braces (Supplemental Fig. 6). In most cases, these produce streak artifacts in the CT images, whereas in the MR images they typically generate an ovoid signal void due to the magnetic susceptibility. Although the CT streak artifacts directly affect the resulting $\mu$ maps, they do not appear in the maps derived using the atlas-based method (Supplemental Fig. 6). However, further studies are required to fully understand the potential benefits and drawbacks of the MR-based approach in this context.

Although mean SUV (and maximum SUV for tumor ROIs) were significantly underestimated for both tumor and reference ROIs when $\mathrm{PET}_{\text {Dixon }}$ was compared with either $\mathrm{PET}_{\mathrm{CT}}$ or $\mathrm{PET}_{\text {atlas}}$, mean or maximum $\mathrm{T} / \mathrm{B}$ also showed a consistent underestimation but just failed to reach statistical significance (Table 2). This could be due to two reasons: first, both reference and tumor ROIs were underestimated for $\mathrm{PET}_{\text {Dixon }}$ in terms of SUV, and therefore by taking their ratio, we could compensate for some (but not all) of the underestimation effects; and second, the small sample of subjects analyzed $(n=7)$, especially considering the first point (a compensation of underestimation factors when the ratio is taken), prevented the T/B from reaching statistical significance. Because bone is not considered in the Dixon approach, highly statistically significant underestimations occur on the $\mathrm{PET}_{\text {Dixon }}$ images that could severely bias the quantification and potential clinical diagnosis.

The results suggest that our method provides more accurate quantification than do previously proposed MR AC methods, in terms of both the accuracy of the $\mu$ maps and PET quantification. Segmentation-based approaches have shown differences (compared with either CT AC or transmission-based AC) of around $10 \%$ or higher for brain imaging $(11,12,28)$. A comparison between a segmentation- and an atlas-based method with 2 different nonrigid algorithms was performed by Malone et al. on 10 control subjects $(8)$. The authors reported that the atlas methods offered better results than transmission-based AC, with mean differences smaller than $2 \%$. Results similar to ours were also presented by Johansson et al. and Larsson et al. with a machine learning method based on a mixture of gaussians $(29,30)$ and by Navalpakkam et al. with a support vector machine (31). The authors obtained accurate LACs, with mean differences in PET reconstructed images of around $2 \%-3 \%$ in both studies compared with CT AC PET image reconstruction. The practical applicability of the methods may be reduced, however, by their requirement for multidimensional training image space including multiple MR images (T2-weighted VIBE and dual-echo ultrashort echo times) in addition to the corresponding CT images. Additionally, these methods relied on similar MR image intensities for different subjects to produce accurate results, which may be challenging across institutions. Kops et al. and Malone et al. previously presented an atlas-method based on the SPM2 nonrigid registration algorithm $(4,8)$. The mean differences reported compared with transmission-based AC revealed an overestimation of $9 \%$. In contrast, our method uses 6 tissue classes instead of intensity images and a diffeomorphic image registration approach, which was already shown to provide accurate results in the brain (26) and is more accurate than the coregistration algorithm included in SPM2 used by Kops et al. (4).

A limitation of our method is that the segmentation of the 6 different classes is less precise in the neck area, and hence the resulting $\mu$ maps are less accurate in this region. This is because the segmentation step relies heavily on prior anatomic information (included in the SPM software) and the New Segment tool in SPM8 is primarily focused on the head, being less accurate in other areas. Fortunately, as only a small fraction of the lines of 
response traverse both the neck area and parts of the brain, the effect on the final PET reconstructed brain images is minimal. The method could in principle be adapted to other body regions, assuming that an accurate segmentation of tissue classes (e.g., bone, soft tissue, fat, air, and lungs) can be performed.

\section{CONCLUSION}

We have presented an SPM8-based approach for deriving the head $\mu$ map from anatomic MR images that could, in principle, be used in any PET/MR scanner. The method combines the strengths of both segmentation- and atlas-based methods while minimizing their drawbacks. Its implementation is straightforward and, unlike other approaches, requires only the data acquired with a single MR sequence (MPRAGE). Compared with the method currently available on the Biograph mMR scanner, PET image quantification is improved by $70 \%$, on average. The improvement is the largest in areas adjacent to cortical bone (e.g., brain cortex), which are of interest in numerous neurologic disorders. A package containing the necessary software to generate the $\mu$ maps from newly acquired MR images will be provided to interested users on request.

\section{DISCLOSURE}

The costs of publication of this article were defrayed in part by the payment of page charges. Therefore, and solely to indicate this fact, this article is hereby marked "advertisement" in accordance with 18 USC section 1734. Funding for this work was provided by NIH grants R01CA137254 and 1R01EB014894. No other potential conflict of interest relevant to this article was reported.

\section{REFERENCES}

1. Catana C, Drzezga A, Heiss WD, Rosen BR. PET/MRI for neurologic applications. J Nucl Med. 2012;53:1916-1925.

2. Bezrukov I, Mantlik F, Schmidt H, Scholkopf B, Pichler BJ. MR-based PET attenuation correction for PET/MR imaging. Semin Nucl Med. 2013;43:45-59.

3. Schulz V, Torres-Espallardo I, Renisch S, et al. Automatic, three-segment, MRbased attenuation correction for whole-body PET/MR data. Eur J Nucl Med Mol Imaging. 2011;38:138-152.

4. Kops ER, Herzog H. Alternative methods for attenuation correction for PET images in MR-PET scanners. Nucl Sci Symp Conf Rec. 2007;6:4327-4330.

5. Hofmann M, Steinke F, Scheel V, et al. MRI-based attenuation correction for PET/MRI: a novel approach combining pattern recognition and atlas registration. J Nucl Med. 2008;49:1875-1883.

6. Beyer T, Weigert M, Quick HH, et al. MR-based attenuation correction for torsoPET/MR imaging: pitfalls in mapping MR to CT data. Eur J Nucl Med Mol Imaging. 2008;35:1142-1146.

7. Hofmann M, Pichler B, Scholkopf B, Beyer T. Towards quantitative PET/MRI: a review of MR-based attenuation correction techniques. Eur J Nucl Med Mol Imaging. 2009;36(suppl 1):S93-S104.

8. Malone IB, Ansorge RE, Williams GB, Nestor PJ, Carpenter TA, Fryer TD. Attenuation correction methods suitable for brain imaging with a PET/MRI scanner: a comparison of tissue atlas and template attenuation map approaches. J Nucl Med. 2011;52:1142-1149.

9. Le Goff-Rougetet R, Frouin V, Mangin J-F, Bendriem B. Segmented MR images for brain attenuation correction in PET. Proc SPIE. 1994;2167:725-736.
10. Martinez-Möller A, Souvatzoglou M, Delso G, et al. Tissue classification as a potential approach for attenuation correction in whole-body PET/MRI: evaluation with PET/CT data. J Nucl Med. 2009;50:520-526.

11. Catana C, van der Kouwe A, Benner T, et al. Toward implementing an MRIbased PET attenuation-correction method for neurologic studies on the MR-PET brain prototype. J Nucl Med. 2010;51:1431-1438.

12. Keereman V, Fierens Y, Broux T, De Deene Y, Lonneux M, Vandenberghe S. MRI-based attenuation correction for PET/MRI using ultrashort echo time sequences. J Nucl Med. 2010;51:812-818.

13. Berker Y, Franke J, Salomon A, et al. MRI-based attenuation correction for hybrid PET/MRI systems: a 4-class tissue segmentation technique using a combined ultrashort-echo-time/Dixon MRI sequence. J Nucl Med. 2012;53:796-804.

14. Nuyts J, Dupont P, Stroobants S, Benninck R, Mortelmans L, Suetens P. Simultaneous maximum a posteriori reconstruction of attenuation and activity distributions from emission sinograms. IEEE Trans Med Imaging. 1999;18:393-403.

15. Salomon A, Goedicke A, Schweizer B, Aach T, Schulz V. Simultaneous reconstruction of activity and attenuation for PET/MR. IEEE Trans Med Imaging. 2011;30:804-813.

16. Rezaei A, Defrise M, Bal G, et al. Simultaneous reconstruction of activity and attenuation in time-of-flight PET. IEEE Trans Med Imaging. 2012;31:22242233.

17. Nuyts J, Bal G, Kehren F, Fenchel M, Michel C, Watson C. Completion of a truncated attenuation image from the attenuated PET emission data. IEEE Trans Med Imaging. 2013;32:237-246.

18. Defrise M, Rezaei A, Nuyts J. Time-of-flight PET data determine the attenuation sinogram up to a constant. Phys Med Biol. 2012;57:885-899.

19. Mollet P, Keereman V, Clementel E, Vandenberghe S. Simultaneous MR-compatible emission and transmission imaging for PET using time-of-flight information. IEEE Trans Med Imaging. 2012;31:1734-1742.

20. Delso G, Furst S, Jakoby B, et al. Performance measurements of the Siemens mMR integrated whole-body PET/MR scanner. J Nucl Med. 2011;52:1914-1922.

21. Sled JG, Zijdenbos AP, Evans AC. A nonparametric method for automatic correction of intensity nonuniformity in MRI data. IEEE Trans Med Imaging. 1998;17:87-97.

22. Burger C, Goerres G, Schoenes S, Buck A, Lonn AH, Von Schulthess GK. PET attenuation coefficients from CT images: experimental evaluation of the transformation of CT into PET 511-keV attenuation coefficients. Eur J Nucl Med Mol Imaging. 2002;29:922-927.

23. Pauleit D, Stoffels G, Bachofner A, et al. Comparison of ${ }^{18}$ F-FET and ${ }^{18}$ F-FDG PET in brain tumors. Nucl Med Biol. 2009;36:779-787.

24. Andersen FL, Ladefoged CN, Beyer T, et al. Combined PET/MR imaging in neurology: MR-based attenuation correction implies a strong spatial bias when ignoring bone. Neuroimage. 2014;84:206-216.

25. Ashburner J. A fast diffeomorphic image registration algorithm. Neuroimage. 2007;38:95-113.

26. Klein A, Andersson J, Ardekani BA, et al. Evaluation of 14 nonlinear deformation algorithms applied to human brain MRI registration. Neuroimage. 2009; 46:786-802.

27. Goerres GW, Ziegler SI, Burger C, Berthold T, Von Schulthess GK, Buck A. Artifacts at PET and PET/CT caused by metallic hip prosthetic material. Radiology. 2003;226:577-584.

28. Samarin A, Burger C, Wollenweber SD, et al. PET/MR imaging of bone lesions: implications for PET quantification from imperfect attenuation correction. Eur $J$ Nucl Med Mol Imaging. 2012;39:1154-1160.

29. Johansson A, Karlsson M, Nyholm T. CT substitute derived from MRI sequences with ultrashort echo time. Med Phys. 2011;38:2708-2714.

30. Larsson A, Johansson A, Axelsson J, et al. Evaluation of an attenuation correction method for PET/MR imaging of the head based on substitute CT images. MAGMA. 2013;26:127-136.

31. Navalpakkam BK, Braun H, Kuwert T, Quick HH. Magnetic resonance-based attenuation correction for PET/MR hybrid imaging using continuous valued attenuation maps. Invest Radiol. 2013;48:323-332. 